\title{
Measuring Estrogen Receptor Functionality Using Progesterone Receptor PET Imaging: Rising to the (Estradiol) Challenge!
}

\author{
Michel Paquette and Éric E. Turcotte \\ Department of Nuclear Medicine and Radiobiology, Faculty of Medicine and Health Sciences, Université de Sherbrooke, Sherbrooke, \\ Québec, Canada
}

See the associated article on page 220.

$\mathbf{P}_{\mathrm{r}}$ rognosis and therapy choice for breast cancer greatly depend on pathohistologic evaluation of biopsy and surgical samples. More specifically, the estrogen receptor (ER)-progesterone receptor (PR)-human epidermal growth factor receptor (HER2) triad provides crucial information on which clinicians guide their intervention. Indeed, positivity for one or several of those receptors indicates that the use of hormone therapy or immunotherapy against their respective targets is likely to benefit the afflicted patient.

However, the success rate of such targeted therapies is far from perfect for receptor-positive diseases, since many known or suspected reasons can explain unforeseen failure of those otherwise efficient and selective treatments. One of the key contributors to this setback is the intra- and intertumor receptor status heterogeneity that can occur in some patients, for which assessment through classic biopsy is suboptimal (1). Another possible reason for endocrine therapy failure is the loss of the associated pathway signaling despite the presence of the receptor it is supposed to target. For example, whereas $70 \%$ of all breast cancers are ERpositive, most of those that respond to ER-targeting endocrine therapy are also PR-positive, the latter having its expression driven by ER (2). Indeed, the hormone therapy response rate was reported in an early study to reach $77 \%$ in ER-positive/PR-positive patients, whereas it plummeted to just 27\% in ER-positive/PR-negative breast cancers (3).

The ER-targeting PET tracer ${ }^{18} \mathrm{~F}-16 \alpha$-fluoroestradiol (FES) enables whole-body imaging in patients and, as such, could palliate erroneous biopsy-derived decisions by better assessing tumor heterogeneity. It was also shown that ${ }^{18} \mathrm{~F}$-FES PET could predict, to a certain extent, the efficacy or failure of hormone therapy $(4,5)$, raising evidence that this imaging modality might be helpful for breast cancer management. One limitation of ${ }^{18} \mathrm{~F}$-FES PET is that it merely reflects the ability of a given ER-positive tumor to bind estradiollike compounds, without giving direct evidence of whether the ER is functional within the tumor.

Received Nov. 6, 2018; revision accepted Nov. 13, 2018.

For correspondence or reprints contact: Éric E. Turcotte, Department of Nuclear Medicine and Radiobiology, Faculty of Medicine and Health Sciences, 3001 12th Ave., Sherbrooke, Québec, Canada J1H 5N4.

E-mail: eric.e.turcotte@usherbrooke.ca

Published online Nov. 21, 2018.

COPYRIGHT (c) 2019 by the Society of Nuclear Medicine and Molecular Imaging. DOI: 10.2967/jnumed.118.220921
To probe ER functionality and at the same time assess whole-body PR status, many PR-targeting PET tracers have been developed, with ${ }^{18} \mathrm{~F}$-fluorofuranylnorprogesterone (FFNP) being the most successful so far (6). Preclinical studies recently suggested that baseline ${ }^{18} \mathrm{~F}-$ FES PET combined with ${ }^{18} \mathrm{~F}$-FFNP PET follow-up could predict response to ER-targeting endocrine therapy (7) and that ${ }^{18} \mathrm{~F}$-FFNP PET is superior to ${ }^{18} \mathrm{~F}$-FDG PET in distinguishing between tumors sensitive to and resistant to estradiol deprivation (8). A vanguard clinical study showed that ${ }^{18} \mathrm{~F}$-FFNP PET enables detection of PR-positive tumors (9). However, ${ }^{18} \mathrm{~F}$-FFNP $\mathrm{SUV}_{\max }$ did not significantly differ between PR-positive and PR-negative lesions; only the tumor-to-nonspecific ratio succeeded in correlating with PR status in patients (9) - a major setback for detection and diagnosis of breast cancer.

In the paper by Salem et al. (10) in this issue of The Journal of Nuclear Medicine, the authors designed an interesting preclinical model with the intention of evaluating the isoform specificity and sensitivity of ${ }^{18}$ F-FFNP. More specifically, they developed a clever PR-A and PR-B overexpression model from the triple-negative cell line MDA-MB-231 to evaluate the isoform specificity of ${ }^{18} \mathrm{~F}-\mathrm{FFNP}$ both in vitro and in vivo. Contrary to what was observed for ${ }^{18} \mathrm{~F}$-FES, which has an approximately 6 -fold preference for ER $\alpha$ over ER $\beta,{ }^{18}$ F-FFNP was shown to target both PR-A and PR-B with similar affinity. ${ }^{18} \mathrm{~F}-\mathrm{FFNP}$ PET in xenografted mice showed a 2-fold higher ${ }^{18} \mathrm{~F}-\mathrm{FFNP}$ uptake in PR-A- and PR-Bexpressing tumors than in PR-negative MDA-MB-231 tumors.

The triple-positive breast cancer cell line T47D was then used both in vitro and in a xenograft model to test the effect of a prior estradiol challenge on cell and tumor ${ }^{18} \mathrm{~F}-\mathrm{FFNP}$ uptake. A strong PR induction was observed using immunohistochemistry and immunoblotting for up to $72 \mathrm{~h}$ after exposure to estradiol. This was accompanied by a significantly and progressively higher ${ }^{18} \mathrm{~F}$-FFNP uptake through time compared with baseline, both in vitro and using a biodistribution assay. This finding is impactful, as it may provide evidence that ${ }^{18}$ F-FFNP PET, used in combination with an estradiol challenge, could be helpful for monitoring whole-body tumor susceptibility to estrogens and, more importantly, to ER-targeting endocrine therapies.

However, one opportunity this study has missed is that no ${ }^{18} \mathrm{~F}$ FFNP PET images of estradiol-stimulated T47D xenografted tumors are displayed. Since T47D ${ }^{18}$ F-FFNP uptake (assessed by biodistribution) was shown to be approximately 8-fold higher than uptake of either PR-A- or PR-B-overexpressing versions of MDA-MB-231 (assessed by PET), ${ }^{18}$ F-FFNP PET of estradiol-induced T47D tumors might have produced images with an exceptionally high tumor contrast. 
Another group already evaluated an estradiol challenge in the clinical setting using a combination of baseline ${ }^{18} \mathrm{~F}-\mathrm{FES}$ PET and posttreatment serial ${ }^{18} \mathrm{~F}$-FDG PET; high ${ }^{18} \mathrm{~F}$-FES tumor uptake combined with a postestrogen increase in ${ }^{18} \mathrm{~F}$-FDG uptake foretold hormone therapy efficacy (11). However, an ${ }^{18}$ F-FDG uptake increase resulting from the hormone-induced metabolic flare was shown to be a better predictor of endocrine therapy efficacy than baseline ${ }^{18} \mathrm{~F}$-FES PET. In this respect, an ${ }^{18} \mathrm{~F}$-FFNP-monitored estradiol challenge might be raised as a much more specific and precise method than serial ${ }^{18} \mathrm{~F}$-FDG PET, as it directly assesses ER transcriptional activity and thus functionality. An ongoing clinical trial by Dr. Farrokh Dehdashti's group at Washington University is evaluating the ${ }^{18} \mathrm{~F}$-FFNP PET/estradiol challenge approach, the results of which, we hope, will unravel the full potential of this protocol to increase the hormone therapy success rate and improve selection of patients more likely to respond.

In summary, this study has shown impressive results, with estradiol stimuli increasing ${ }^{18} \mathrm{~F}$-FFNP uptake in T47D tumors to approximately $12 \%$ injected dose/g at $72 \mathrm{~h}$ after challenge, which is unprecedented for steroid-based tracers in the preclinical setting. If this preclinical study is a portent of how well this estradiol challenge protocol will work in the clinical setting, we might have here a preview of what could become an indispensable tool to better use and predict the efficacy of endocrine therapy.

\section{DISCLOSURE}

No potential conflict of interest relevant to this article was reported.

\section{REFERENCES}

1. Zardavas D, Irrthum A, Swanton C, Piccart M. Clinical management of breast cancer heterogeneity. Nat Rev Clin Oncol. 2015;12:381-394.

2. Bardou VJ, Arpino G, Elledge RM, Osborne CK, Clark GM. Progesterone receptor status significantly improves outcome prediction over estrogen receptor status alone for adjuvant endocrine therapy in two large breast cancer databases. J Clin Oncol. 2003;21:1973-1979.

3. Osborne CK, Yochmowitz MG, Knight WA, McGuire WL. The value of estrogen and progesterone receptors in the treatment of breast cancer. Cancer. 1980;46: 2884-2888.

4. Kurland BF, Peterson LM, Lee JH, et al. Estrogen receptor binding (FES PET) and glycolytic activity (FDG PET) predict progression-free survival on endocrine therapy in patients with ER+ breast cancer. Clin Cancer Res. 2017;23:407-415.

5. van Kruchten M, de Vries EG, Glaudemans AW, et al. Measuring residual estrogen receptor availability during fulvestrant therapy in patients with metastatic breast cancer. Cancer Discov. 2015;5:72-81.

6. Buckman BO, Bonasera TA, Kirschbaum KS, Welch MJ, Katzenellenbogen JA. Fluorine-18-labeled progestin 16 alpha, 17 alpha-dioxolanes: development of highaffinity ligands for the progesterone receptor with high in vivo target site selectivity. J Med Chem. 1995;38:328-337.

7. Fowler AM, Chan SR, Sharp TL, et al. Small-animal PET of steroid hormone receptors predicts tumor response to endocrine therapy using a preclinical model of breast cancer. J Nucl Med. 2012;53:1119-1126.

8. Chan SR, Fowler AM, Allen JA, et al. Longitudinal noninvasive imaging of progesterone receptor as a predictive biomarker of tumor responsiveness to estrogen deprivation therapy. Clin Cancer Res. 2015;21:1063-1070.

9. Dehdashti F, Laforest R, Gao F, et al. Assessment of progesterone receptors in breast carcinoma by PET with $21-{ }^{18}$ F-fluoro- $16 \alpha, 17 \alpha-\left[(R)-\left(1^{\prime}-\alpha\right.\right.$-furylmethylidene) dioxy]-19-norpregn-4-ene-3,20-dione. J Nucl Med. 2012;53:363-370.

10. Salem K, Kumar M, Yan Y, et al. Sensitivity and isoform specificity of ${ }^{18} \mathrm{~F}$ fluorofuranylnorprogesterone for measuring progesterone receptor protein response to estradiol challenge in breast cancer. J Nucl Med. 2019;60:220-226.

11. Dehdashti F, Mortimer JE, Trinkaus K, et al. PET-based estradiol challenge as a predictive biomarker of response to endocrine therapy in women with estrogenreceptor-positive breast cancer. Breast Cancer Res Treat. 2009;113:509-517. 